\title{
A GENERALIZATION OF A THEOREM BY HARDY AND LITTLEWOOD
}

\author{
A. C. ALLEN
}

1. Suppose that $\phi(x)$ is non-negative and integrable in $(0,1)$, so that it is measurable and finite almost everywhere. If $M(y)$ is the measure of the set in which $\phi(x) \geqq y, M(y)$ is a decreasing function of $y$. The inverse $\Phi$ of $M$ is defined by

$$
\Phi\{M(y)\}=y,
$$

and $\phi(x)$ is a decreasing function of $x$ defined uniquely in $(0,1)$ except for at most an enumerable set of values of $x$, viz., those corresponding to intervals of constancy of $M(y)$. We may complete the definition of $\phi(x)$ by agreeing, for example, that

$$
\Phi(x)=\{\phi(x-0)+\Phi(x+0)\} / 2
$$

at a point of discontinuity.

We call $\phi(x)$ the rearrangement of $\phi(x)$ in decreasing order.

2. The following theorem which is important for its functiontheoretic applications is due to Hardy and Littlewood [1]. The theorem may be stated in two equivalent forms.

Theorem A. Suppose that $f(x)$ is non-negative and integrable in $(0,1)$, that

$$
\Theta(x)=\Theta(x, f)=\max _{0 \leq \xi<x} \frac{1}{x-\xi} \int_{\xi}^{x} f(t) d t .
$$

Then

$$
\bar{\Theta}(x) \leqq \frac{1}{x} \int_{0}^{x} \bar{f}(t) d t
$$

for $0<x \leqq 1$.

Theorem B. Suppose that $f(x)$ satisfies the conditions of Theorem A, and that $s(y)$ is any increasing function of $y$ defined for $y \geqq 0$. Then

$$
\int_{0}^{1} s\{\Theta(x)\} d x \leqq \int_{0}^{1} s\left\{\frac{1}{x} \int_{0}^{x} \bar{f}(t) d t\right\} d x
$$

In this note I generalize the definition of $\Theta(x)$, and prove theorems analogous to those above.

Received by the editors January 11, 1952. 
3. Suppose that $k(t)$ is a non-negative, decreasing function of $t$, defined and integrable in $(0,1)$.

THEOREM 1. Suppose that $f(x)$ is non-negative and integrable in $(0,1)$, that $k(t)$ satisfies the conditions of the last paragraph, that $k(t) \bar{f}(t)$ is integrable in $(0,1)$, and that

$$
\Theta(x)=\Theta(x, f)=\max _{0<h \leqq x} \frac{1}{h} \int_{0}^{h} k\left(\frac{t}{h}\right) f(x-h+t) d t .
$$

Then

$$
\bar{\Theta}(x) \leqq \frac{1}{x} \int_{0}^{x} k\left(\frac{t}{x}\right) \bar{f}(t) d t
$$

for $0<x \leqq 1$.

Theorem 2. Suppose that $f(x)$ satisfies the conditions of Theorem 1, and that $s(y)$ is any increasing function of $y$ defined for $y \geqq 0$. Then

$$
\int_{0}^{1} s\{\Theta(x)\} d x \leqq \int_{0}^{1} s\left\{\frac{1}{x} \int_{0}^{x} k\left(\frac{t}{x}\right) \bar{f}(t) d t\right\} d x .
$$

The equivalence of Theorems 1 and 2 may be proved in the same way as is used to prove the equivalence of Theorems $A$ and $B$ $[2,10.18]$.

It is easily seen that Theorems A and B are the particular case of Theorems 1 and 2 where $k(t)$ is the kernel of the first Cesaro mean, $(C, 1)$. The latter theorems will also deal with the case $(C, \delta)$, $0<\delta \leqq 1$.

In this paper I prove Theorem 2, from which Theorem 1 may be deduced. Firstly, we shall prove Theorem 2 for the special case where

$$
s(t)=s_{\alpha}(t)= \begin{cases}0 & \text { for } 0 \leqq t \leqq \alpha, \\ 1 & \text { for } t>\alpha .\end{cases}
$$

In this case, if we rewrite Theorem 2 , it is required to prove that

$$
\underset{0 \leqq x \leqq 1}{m E}[x, \Theta(x)>\alpha] \leqq \underset{0 \leqq x \leqq 1}{m E}\left[x, \frac{1}{x} \int_{0}^{x} k\left(\frac{t}{x}\right) \bar{f}(t) d t>\alpha\right] .
$$

We require the following lemmas:

Lemma 1. Suppose that each point of a set in $(a, b)$ is the right-hand end point of one or more intervals $\left(x-h_{x}, x\right)$ of a family $H$. Then there is a finite nonoverlapping set $S$ of intervals of $H$ which includes a subset $E_{\text {! }}$ of $E$ such that $m E_{\text {! }}>m E-\epsilon$. 
This result is due to Sierpinski [3].

Lемма 2. If $f(x)$ is non-negative and integrable in $(0, a+b), k(t)$ is a non-negative, decreasing function of $t$ in $(0,1), k(t) \bar{f}(t)$ is integrable, and if

$$
\frac{1}{a} \int_{0}^{a} k\left(\frac{t}{a}\right) f(t) d t>\alpha, \quad \frac{1}{b} \int_{0}^{b} k\left(\frac{t}{b}\right) f(t+a) d t>\alpha,
$$

then

$$
\frac{1}{a+b} \int_{0}^{a+b} k\left(\frac{t}{a+b}\right) \bar{f}(t) d t>\alpha
$$

Since $k(t)$ is decreasing and integrable in $(0,1)$, it has a non-negative derivative almost everywhere. Also, writing

$$
F(x)=\int_{0}^{x} f(t) d t
$$

and from (4), integrating by parts, we have

$$
\begin{gathered}
k(1) F(a)+\int_{0}^{1} k^{\prime}(t) F(a t) d t>a \alpha \\
k(1)\{F(a+b)-F(a)\}+\int_{0}^{1} k^{\prime}(t)\{F(a+b t)-F(a)\} d t>b \alpha .
\end{gathered}
$$

Adding, we have

(5) $k(1) F(a+b)+\int_{0}^{1} k^{\prime}(t)\{F(a t)+F(a+b t)-F(a)\} d t>(a+b) \alpha$.

Similarly, we have

$$
\begin{aligned}
& \int_{0}^{a+b} k\left(\frac{t}{a+b}\right) \bar{f}(t) d t \\
&=k(1) \int_{0}^{a+b} \bar{f}(t) d t+\int_{0}^{1} k^{\prime}(t)\left\{\int_{0}^{(a+b) t} \bar{f}(s) d s\right\} d t \\
&=k(1) F(a+b)+\int_{0}^{1} k^{\prime}(t)\left\{\int_{0}^{(a+b) t} \bar{f}(s) d s\right\} d t
\end{aligned}
$$

Now it is evident from the definition of $\bar{f}(t)$ that, when $m E=(a+b) t$,

$$
\int_{0}^{(a+b) t} \bar{f}(s) d s \geqq \int_{E} f(s) d s .
$$


Hence,

$$
\int_{0}^{(a+b) t} \bar{f}(s) d s \geqq F(a t)+F(a+b t)-F(a),
$$

and, since almost everywhere $k^{\prime}(t) \geqq 0$, we have

$$
\begin{aligned}
\int_{0}^{a+b} k\left(\frac{t}{a+b}\right) & \bar{f}(t) d t \geqq k(1) F(a+b) \\
& +\int_{0}^{1} k^{\prime}(t)\{F(a t)+F(a+b t)-F(a)\} d t>(a+b) \alpha,
\end{aligned}
$$

from which the result follows.

4. We are now in a position to prove (3).

From the definition of $\Theta(x)$ it is seen that to every point $x$ of $E_{0 \leqq x \leqq 1}[x, \Theta(x)>\alpha]$ there exists at least one interval $\left(x-h_{x}, x\right)$ $\subset(0,1)$ for which

$$
\frac{1}{h_{x}} \int_{0}^{h_{x}} k\left(\frac{t}{h_{x}}\right) f\left(x-h_{x}+t\right) d t>\alpha .
$$

Let us apply Lemma 1 to the set $E$. Then there exists a finite set $S$ of the above intervals which are nonoverlapping, which cover a subset $E_{\epsilon}^{\prime}$ of $E$ such that $m E_{\epsilon}^{\prime}>m E-\epsilon$. It is evidently sufficient to prove (3) when we replace $m E$ in the left-hand side by $m E_{e}^{\prime}$ for all $\epsilon>0$, and, a fortiori, it is sufficient to prove that

$$
m S \leqq \underset{0 \leqq x \leqq 1}{m E}\left[x, \frac{1}{x} \int_{0}^{x} k\left(\frac{t}{x}\right) \bar{f}(t) d t>\alpha\right] .
$$

We further use the legitimate simplification that $f(t)=0$ for $t$ in the complement of $S$, since this decreases the right-hand side of (6). This latter assumption permits us to translate the intervals of $S$ to the left until they are end to end, and completely cover the interval $(0, m S)$, i.e., we have the $n$ intervals $\left(j_{r}, j_{r+1}\right)$ with $0=j_{0} \leqq j_{r}<j_{r+1} \leqq j_{n}$ $=m S$.

Consider the two intervals $\left(j_{n-2}, j_{n-1}\right),\left(j_{n-1}, j_{n}\right)$.

We have

$$
\begin{array}{r}
\frac{1}{j_{n}-j_{n-1}} \int_{0}^{j_{n}-\jmath_{n-1}} k\left(\frac{t}{j_{n}-j_{n-1}}\right) f(t) d t>\alpha, \\
\frac{1}{j_{n-1}-j_{n-2}} \int_{0}^{j_{n-1}-j_{n-2}} k\left(\frac{t}{j_{n-1}-j_{n-2}}\right) f(t) d t>\alpha .
\end{array}
$$


Let $f_{1}(t)$ be the decreasing rearrangement of $f(t)$ within the above two intervals. Then, applying Lemma 2 , we have

$$
\frac{1}{j_{n}-j_{n-2}} \int_{0}^{j_{n}-j_{n-2}} k\left(\frac{t}{j_{n}-j_{n-2}}\right) f_{1}(t) d t>\alpha .
$$

We have thus reduced the case from $n$ intervals to $(n-1)$ intervals. Repeating the process a further $(n-2)$ times we arrive at $\bar{f}(t)$ defined in the single interval $(0, m S)$, and have

$$
\frac{1}{m S} \int_{0}^{m S} k\left(\frac{t}{m S}\right) \bar{f}(t) d t>\alpha
$$

which proves (6).

Thus we have proved the theorem for the special case (2).

5. Since $s(t)$ is an increasing function of $t$, we may approximate to it uniformly in any finite interval $(0, N)$ by the sum of a finite number of functions of the type $s_{\alpha}(t)$. The deduction of Theorem 2 from the special case considered above is apparent.

The author is indebted to the Commonwealth Fund of New York for a Commonwealth Fellowship.

\section{BIBLIOGRAPHY}

1. Hardy and Littlewood, $A$ maximal theorem with function-theoretic applications, Acta Math. vol. 54 (1930) pp. 81-116.

2. Hardy, Littlewood, and P6lya, Inequalities, Cambridge, 1934.

3. Sierpinski, Un lemme metrique, Fund. Math. vol. 4 (1923) pp. 201-203.

Princeton University 